\title{
Quorum of observables for universal quantum estimation
}

\author{
G. Mauro D'Ariano, Lorenzo Maccone and Matteo G. A. Paris \\ QuAntum Optics Group, INFM at Dipartimento di Fisica 'Alessandro Volta' \\ Università degli Studi di Pavia, via Bassi 6, I-27100 Pavia, Italy
}

\begin{abstract}
Any method for estimating the ensemble average of arbitrary operator (observables or not, including the density matrix) relates the quantity of interest to a complete set of observables, i.e. a quorum. This corresponds to an expansion on an irreducible set of operators in the Liouville space. We give two general characterizations of these sets. All the known unbiased reconstruction techniques, i.e. "quantum tomographies", can be described in this framework. New operatorial resolutions are given that can be used to implement novel reconstruction schemes.
\end{abstract}

\section{Introduction}

In order to characterize a quantum system, one can measure an observable, or a set of observables, on repeated identical preparations of the system. A question immediately arises: is this set a quorum, i.e. is it sufficient to give a complete quantum information on the system? In other words, is it possible to estimate the expectation values of any system operator? This issue is of a great practical interest for fundamental experiments in quantum measurement theory, as well as for a potential application as a quantum standard in the new field of quantum information [1].

Different estimation techniques have been proposed tailored to different systems, such as the radiation field [2], trapped ions and molecular vibrational states [3], spin systems [4], and a unified approach is desirable. For set of operators that exhibit a group symmetry, a general theory, the so called "group tomography", has been established [5]. However, not all estimation techniques can be described within a group-theoretical scheme, and the purpose of this paper is to give a more general framework. As we will see, all the known quantum estimation techniques can be embodied in the present approach. In addition, the formalism here presented is of help for the derivation of new operatorial resolutions that result into novel estimation techniques.

The paper is structured as follows. In section 2, we establish the general conditions for a quorum of observables. Sections 6, 4, and 5 are devoted to examples of the estimation technique, for the harmonic oscillator, general spin systems, and the free particle respectively. Section 6 closes the paper with a summary. We added Appendix 
A to reformulate the theory in the familiar Dirac formalism, and Appendix B to give a constructive algorithm to derive tomographic basis for finite-dimensional Hilbert space.

\section{Quantum estimation}

The indirect (tomographic) reconstruction 用 of an operator $A$ is possible when there exists a resolution of the form

$$
\hat{A}=\int_{\mathcal{X}} d x \operatorname{Tr}\left[\hat{A} \hat{B}^{\dagger}(x)\right] \hat{C}(x)
$$

where $x$ is a (possibly multidimensional) parameter living on a (discrete or continuous) manifold $\mathcal{X}$. The only hypothesis in (11) is the existence of the trace. The operators $\hat{C}(x)$ are functions of the quorum of observables measured for the reconstruction, whereas the operators $\hat{B}(x)$ form the dual basis of the set $\hat{C}(x)$. The term

$$
\mathcal{E}[\hat{A}](x)=\operatorname{Tr}\left[\hat{A} \hat{B}^{\dagger}(x)\right] \hat{C}(x)
$$

represents the quantum estimator for the operator $\hat{A}$. Indeed, the expectation value of $\hat{A}$, namely the quantity of interest, is given by the ensemble average

$$
\langle\hat{A}\rangle \doteq \operatorname{Tr}[\hat{A} \hat{\varrho}]=\int_{\mathcal{X}} d x \operatorname{Tr}\left[\hat{A} \hat{B}^{\dagger}(x)\right] \operatorname{Tr}[\hat{C}(x) \hat{\varrho}] \equiv \int_{\mathcal{X}} d x\langle\mathcal{E}[\hat{A}](x)\rangle
$$

where $\varrho$ is the density matrix of the quantum system under investigation. The averaged estimator in Eq. (3) is the product of two terms: The quantity $\operatorname{Tr}[\hat{C}(x) \hat{\varrho}]$ depends only on the quantum state, and it is related to the probability distribution of the measurement outcomes, whereas the term $\operatorname{Tr}\left[\hat{A} \hat{B}^{\dagger}(x)\right]$ depends only on the quantity to be measured. In particular, the tomography of the quantum state of a system corresponds to writing Eq. (11) for the operators $\hat{A}=|k\rangle\langle n|,\{|n\rangle\}$ being a given Hilbert space basis. For a given system, the existence of a set of operators $\hat{C}(x)$, together with its dual basis $\hat{B}(x)$ allows universal quantum estimation, i. e. the reconstruction of any operator.

We now give two characterizations of the sets $\hat{B}(x)$ and $\hat{C}(x)$ that are necessary and sufficient conditions for writing Eq. (11).

\section{Condition 1: bi-orthogonality}

Let us consider a complete orthonormal set $|n\rangle(n=0,1, \cdots)$. Formula (1) is equivalent to the bi-orthogonality condition

$$
\int_{\mathcal{X}} d x\left\langle q\left|\hat{B}^{\dagger}(x)\right| p\right\rangle\langle m|\hat{C}(x)| l\rangle=\delta_{m p} \delta_{l q},
$$

where $\delta_{i j}$ is the Kronecker delta. Eq. (代) can be straightforwardly generalized to a continuous basis.

$\ddagger$ Among the existing estimation techniques for quantum systems, the so-called quantum homodyne tomography of a single radiation mode has received much attention in the literature [3, 8. In this paper, the term "tomography" is collectively used to denote any kind of state-reconstruction technique. 
Quorum of observables for universal quantum estimation

\section{Condition 2: completeness}

If the set of operators $\hat{C}(x)$ is irreducible, namely if any operator can be written as a linear combination of the $\hat{C}(x)$ as

$$
\hat{A}=\int_{\mathcal{X}} d x a(x) \hat{C}(x)
$$

then Eq. (1) is also equivalent to the trace condition

$$
\operatorname{Tr}\left[\hat{B}^{\dagger}(x) \hat{C}(y)\right]=\delta(x, y)
$$

where $\delta(x, y)$ is a reproducing kernel for the set $\hat{B}(x)$, namely it is a function or a tempered distribution which satisfies

$$
\int_{\mathcal{X}} d x \hat{B}(x) \delta(x, y)=\hat{B}(y)
$$

One can easily see that an analogous identity holds for the set of $C(x)$

$$
\int_{\mathcal{X}} d x \hat{C}(x) \delta(x, y)=\hat{C}(y)
$$

The proofs are straightforward. The irreducibility condition on the operators $\hat{C}(x)$ is essential for the equivalence of (1) and (6). A simple counterexample is provided by the set of projectors $\hat{P}(x)=|x\rangle\langle x|$ over the eigenstates of a selfadjoint operator $\hat{X}$. In fact, Eq. (6) is satisfied by $P(x)$. However, since they do not form an irreducible set, it is not possible to express a generic operator as $\hat{O} \neq \int_{\mathcal{X}} d x\langle x|\hat{O}| x\rangle|x\rangle\langle x|$.

If either the set $\hat{B}(x)$ or the set $\hat{C}(x)$ satisfy the additional trace condition

$$
\begin{aligned}
& \operatorname{Tr}\left[\hat{B}^{\dagger}(y) \hat{B}(x)\right]=\delta(x, y) \\
& \operatorname{Tr}\left[\hat{C}^{\dagger}(y) \hat{C}(x)\right]=\delta(x, y),
\end{aligned}
$$

then we have $\hat{C}(x)=\hat{B}(x)$ (notice that neither $\hat{B}(x)$ nor $\hat{C}(x)$ need to be unitary). In this case, Eq. (1) may be rewritten as

$$
\hat{A}=\int_{\mathcal{X}} d x \operatorname{Tr}\left[\hat{A} \hat{C}^{\dagger}(x)\right] \hat{C}(x) .
$$

In abstract terms a certain number of observables $\hat{Q}_{x}$ constitute a quorum when there are functions $f_{x}\left(\hat{Q}_{x}\right)=\hat{C}(x)$ such that $C(x)$ form an irreducible set Notice that if a set of observables $\hat{Q}_{x}$ constitutes a quorum, than the set of projectors $|q\rangle_{x x}\langle q|$ over their eigenvectors provides a quorum too, with the measure $d x$ in Eq. (1) including the measure $d q$. Of course it is of interest to connect a quorum of observables to a resolution of the form (11), since only in this case there can be a feasible reconstruction scheme. If a resolution formula is written in terms of a set of selfadjoint operators, the set itself constitutes the desired quorum. However, in general a quorum of observables is functionally connected to the corresponding resolution formula. If the operators $\hat{C}(x)$ are unitary, then they can always be considered as exponential of a set of selfadjoint operators, say $\hat{Q}_{x}$. The quantity $\operatorname{Tr}[\hat{C}(x) \hat{\varrho}]$ is thus connected with the 
moment generating function of the set $\hat{Q}_{x}$, and hence to the probability density $p(q ; x)$ of the measurement outcomes, which play the role of the Radon transform the quantum tomography of the harmonic oscillator [6]. Here, the basic resolution formula involves the set of displacement operators $\hat{D}(\alpha)=\exp \left[\alpha a^{\dagger}-\bar{\alpha} a\right]$, which may be viewed as exponential of the field-quadrature operators $\hat{x}_{\phi}=\frac{1}{2}\left(a e^{-i \phi}+a^{\dagger} e^{i \phi}\right)$. In general, the operators $\hat{C}(x)$ can be any function (neither self-adjoint nor unitary) of observables and, even more generally, they may be connected to POVMs rather than observables. In Sections 3.3 and 3.4 we will see examples of this situation.

\section{Quantum estimation for harmonic system}

The harmonic oscillator (HO) model provides a detailed description of several systems of interest in quantum mechanics, as the vibrational states of molecules, the motion of an ion in a Paul trap, and a single mode radiation field. Different proposals have been suggested in order to reconstruct the quantum state of a harmonic system. As we will see in the following they can be summarized using the framework of the previous section, which is also useful for devising novel estimation techniques.

\subsection{Quantum homodyne tomography}

Perhaps the most famous quantum estimation technique is given by the so-called quantum homodyne tomography [7]. Homodyne tomography applies to a single-mode radiation field as well as to the vibrational state of a molecule or a trapped ion, and consists of a set of repeated measurements of the quadrature operator $\hat{q}_{\phi}=$ $\frac{1}{2}\left(a e^{-i \phi}+a^{\dagger} e^{i \phi}\right)$ at different values of the reference phase $\phi$ \$. For homodyne tomography, the relevant operatorial resolution is provided by the set of (irreducible) displacement operators $\hat{D}(\alpha)=\exp \left(\alpha a^{\dagger}-\bar{\alpha} a\right), \alpha \in \mathbb{C}$. In fact, for the displacements Eqs. (6) and (10) hold since

$$
\operatorname{Tr}\left[\hat{D}(\alpha) \hat{D}^{\dagger}(\beta)\right]=\pi \delta^{(2)}(\alpha-\beta),
$$

and Eq. (11) reduces to the Glauber formula

$$
\hat{A}=\int_{\mathbb{C}} \frac{d^{2} \alpha}{\pi} \operatorname{Tr}\left[\hat{A} \hat{D}^{\dagger}(\alpha)\right] \hat{D}(\alpha) .
$$

Changing to polar variables $\alpha=(i / 2) k e^{i \phi}$, Eq. (13) becomes 8

$$
\hat{A}=\int_{0}^{\pi} \frac{d \phi}{\pi} \int_{-\infty}^{+\infty} \frac{d k|k|}{4} \operatorname{Tr}\left(\hat{A} e^{i k \hat{q}_{\phi}}\right) e^{-i k \hat{q}_{\phi}},
$$

which shows explicitly the dependence on the quorum $\hat{q}_{\phi}$. After taking the ensemble average of both members, evaluating this trace over the set of eigenvectors of $\hat{q}_{\phi}$, one obtains

$$
\langle\hat{A}\rangle=\int_{0}^{\pi} \frac{d \phi}{\pi} \int_{-\infty}^{+\infty} d q p(q ; \phi) R[\hat{A}](q ; \phi),
$$

$\S$ For the vibrational tomography the quadrature operator is a time-evolved position or momentum. 
where $p(q ; \phi)={ }_{\phi}\langle q|\hat{\varrho}| q\rangle_{\phi}$ is the probability distribution of quadratures outcomes. The tomographic kernel for the operator $\hat{A}$ is given by

$$
R[\hat{A}](q ; \phi)=\operatorname{Tr}\left[\hat{A} K\left(q-\hat{q}_{\phi}\right)\right],
$$

where the integral kernel $K(z)$ is

$$
K(z)=-\frac{1}{2} \mathrm{P} \frac{1}{z^{2}} \equiv-\lim _{\varepsilon \rightarrow 0^{+}} \frac{1}{2} \operatorname{Re} \frac{1}{(z+i \varepsilon)^{2}},
$$

$\mathrm{P}$ denoting the Cauchy principal value.

Using our condition (6) one can see that the Glauber formula can be generalized to

$$
\hat{A}=\int_{\mathbb{C}} \frac{d^{2} \alpha}{\pi} \operatorname{Tr}\left[\hat{A} \hat{F}_{1} \hat{D}(\alpha) \hat{F}_{2}\right] \hat{F}_{2}^{-1} \hat{D}^{\dagger}(\alpha) \hat{F}_{1}^{-1}
$$

where $\hat{F}_{1}$ and $\hat{F}_{2}$ are two generic invertible operators. By choosing $\hat{F}_{1}^{\dagger}=\hat{F}_{2}=\hat{S}(\zeta)$ with

$$
\hat{S}(\zeta)=\exp \left[\frac{1}{2}\left(\zeta^{2} a^{\dagger 2}-\bar{\zeta}^{2} a^{2}\right)\right] \quad \zeta \in \mathbb{C}
$$

the squeezing operator, we arrive to a different tomographic resolution

$$
\langle\hat{A}\rangle=\int_{0}^{\pi} \frac{d \phi}{\pi} \int_{-\infty}^{+\infty} d q p_{\zeta}(q ; \phi) \operatorname{Tr}\left[\hat{A} \hat{K}\left[q-\hat{q}_{\phi \zeta}\right]\right],
$$

in terms of the probability distribution of the generalized squeezed quadrature operators

$$
\begin{aligned}
\hat{q}_{\phi \zeta} & =\hat{S}^{\dagger}(\zeta) \hat{q}_{\phi} \hat{S}(\zeta) \\
& =\frac{1}{2}\left[\left(\mu e^{i \phi}+\nu e^{-i \phi}\right) a^{\dagger}+\left(\mu e^{-i \phi}+\bar{\nu} e^{i \phi}\right) a\right],
\end{aligned}
$$

where $\mu=\cosh |\zeta|$ and $\nu=\sinh |\zeta| \exp (2 i \arg [\zeta])$. Such an estimation technique has been investigated in detail in Ref. [10].

\subsection{Phase-space estimation techniques}

A different estimation technique based on the generalized Glauber formula (18) may be obtained by putting $\hat{F}_{1}=\hat{I}$, the identity operator, and $\hat{F}_{2}=(-)^{a^{\dagger} a}$, the parity operator. In this case one gets

$$
\hat{A}=\int_{\mathbb{C}} \frac{d^{2} \alpha}{\pi} \operatorname{Tr}\left[\hat{A} \hat{D}^{\dagger}(\alpha)(-)^{a^{\dagger} a}\right](-)^{a^{\dagger} a} \hat{D}(\alpha),
$$

and therefore changing variable to $\alpha=2 \beta$ and using the relation $(-)^{a^{\dagger} a} \hat{D}(2 \beta)=$ $\hat{D}^{\dagger}(\beta)(-)^{a^{\dagger}} \hat{D}(\beta)$

$$
\langle\hat{A}\rangle=\int_{\mathbb{C}} \frac{d^{2} \beta}{\pi} \operatorname{Tr}\left[\hat{A} 4 \hat{D}^{\dagger}(\beta)(-)^{a^{\dagger} a} \hat{D}(\beta)\right] \operatorname{Tr}\left[\hat{D}(\beta) \hat{\varrho} \hat{D}^{\dagger}(\beta)(-)^{a^{\dagger} a}\right] .
$$

Eq. (24) says that it is possible to estimate a $\mathrm{HO}$ operator $\hat{A}$ by repeated measurement of the parity operator on displaced versions of the state under investigation. An approximated implementation of this technique for a single mode radiation field has been suggested [11, 12] through the measurement of the photon number probability on signals displaced by means of a beam splitter. A similar schemes has been used for 
the experimental determination of the motional quantum state of a trapped atom [13]. The advantage of (24) compared to the approximated methods is in the possibility of directly obtaining the kernel $K[\hat{A}](\alpha)$ for any operator $\hat{A}$ for which the trace exists. For instance, the reconstruction of the density matrix in the Fock representation may be obtained by averaging the kernel

$$
\begin{aligned}
K[|n\rangle\langle n+d||](\alpha) & =\left\langle n+d\left|4 \hat{D}^{\dagger}(\alpha)(-)^{a^{\dagger} a} \hat{D}(\alpha)\right| n\right\rangle \\
& =4(-)^{n+d} \exp \left\{-2|\alpha|^{2}\right\} \sqrt{\frac{n !}{(n+d) !}}(2 \alpha)^{d} L_{n}^{d}\left(4|\alpha|^{2}\right),
\end{aligned}
$$

without the need of artificial cut-off in the Fock space [13.

\subsection{Nonlinear phase tomography}

Let us now consider the set of selfadjoint operators given by

$$
\hat{B}(\psi, \phi)=\hat{V}^{\dagger}(\psi) \mu(\phi) \hat{V}(\psi) \quad \psi, \phi \in[0,2 \pi),
$$

where $\mu(\phi)$ is the canonical London' POVM describing the ideal measurement of the HO phase and $\hat{V}(\psi)$ describes a nonlinear phase-shift, namely

$$
\begin{aligned}
& \mu(\phi)=\left|e^{i \phi}\right\rangle\left\langle e^{i \phi}\right| \\
& \hat{V}(\psi)=\exp \left[i\left(a^{\dagger} a\right)^{2} \psi\right],
\end{aligned}
$$

where $\left|e^{i \phi}\right\rangle=\sum_{n} e^{i n \phi}|n\rangle$ is the Susskind-Glogower vector. For a single-mode radiation field the action of $\hat{V}(\psi)$ corresponds to a nonlinear Kerr interaction, whereas for a trapped ion it could be obtained by laser excitation of vibronic levels [14].

One may argue that the measurement of the operators $\hat{B}(\psi, \phi)$ provides a complete characterization of the state under investigation. This is indeed the case with $B(\psi, \phi)$ as a self-dual basis, as it can be easily proved using the bi-orthogonality condition (《)

$$
\int_{0}^{2 \pi} \int_{0}^{2 \pi} \frac{d \phi}{2 \pi} \frac{d \psi}{2 \pi}\langle m|\hat{B}(\psi, \phi)| l\rangle\langle q|\hat{B}(\psi, \phi)| p\rangle=\delta_{p^{2}-q^{2}, m^{2}-l^{2}} \delta_{p-q, l-m}=\delta_{m p} \delta_{l q} .
$$

The kernel for the operator $\hat{A}$ is obtained from equation (3)

$$
K[\hat{A}](\phi, \psi)=\operatorname{Tr}[\hat{A} \hat{B}(\phi, \psi)] .
$$

In particular, the kernel $K\left[\hat{P}_{n d}\right](\phi, \psi)$ for the matrix elements is given by

$$
K\left[\hat{P}_{n d}\right](\phi, \psi)=\langle n+d|\hat{B}(\phi, \psi)| n\rangle=\exp \left[i \psi\left(d^{2}+2 n d\right)+i \phi d\right] .
$$

Notice that, for diagonal matrix elements, Eq. (31) needs a regularization procedure. In fact, the kernel for the projector $\hat{P}_{n n}$ is given by

$$
K_{\varepsilon}\left[\hat{P}_{n n}\right](\phi, \psi)=\exp [i 2 \psi n \varepsilon+i \phi \varepsilon],
$$

with the limit $\varepsilon \rightarrow 0$ that should be taken after the average over the probability density $p(\phi, \psi)=\operatorname{Tr}[\hat{\varrho} \hat{B}(\phi, \psi)]$. A similar procedure should be employed for the reconstruction of any operator which is a function of the number operator $a^{\dagger} a$ only. 
The nonlinear "phase tomography" presented in this section represents a novel resolution formula for harmonic oscillator operators. The corresponding reconstruction technique is based on an ideal phase measurement of the Kerr-displaced state. Hence, in this scheme the quorum is a POVM.

\subsection{A nonunitary resolution formula}

In this section we present a resolution formula in term of nonunitary operators and show how its implementation would correspond to a generalized measurement i.e. a POVM. The operators $\hat{R}_{n}(\phi)$ with $n \in \mathbb{Z}$ and $\phi \in[0,2 \pi)$ are defined as follows

$\hat{R}_{n}(\phi)=\left\{\begin{array}{cc}e_{+}^{n} e^{i a^{\dagger} a \phi} & n \geq 0 \\ e_{-}^{-n} e^{i a^{\dagger} a \phi} & n \leq 0\end{array} \quad \hat{R}_{n}^{\dagger}(\phi)=\left\{\begin{array}{cc}e^{-i a^{\dagger} a \phi} e_{-}^{n} & n \geq 0 \\ e^{-i a^{\dagger} a \phi} e_{+}^{-n} & n \leq 0\end{array}\right.\right.$

where $e_{-}=\sum_{n}|n+1\rangle\left\langle n\left|, e_{+}=\sum_{n}\right| n\right\rangle\langle n+1|$ are the so-called lowering and raising operators for the harmonic oscillator. The operators $\hat{R}_{n}(\phi)$ are not unitary, however $\hat{R}_{n}^{\dagger}(\phi)$ plays the role of dual basis for $\hat{R}_{n}(\phi)$. This can be easily seen using condition (6), for instance we have

$$
\begin{aligned}
\operatorname{Tr}\left[\hat{R}_{k}^{\dagger}(\psi) \hat{R}_{n}(\phi)\right] \stackrel{n, k>0}{=} \operatorname{Tr}\left[e^{-i a^{\dagger} a \psi} e_{-}^{k} e_{+}^{n} e^{i a^{\dagger} a \phi}\right] \\
=\sum_{p} e^{i p(\phi-\psi)} \delta_{p+n, p+k}=\delta_{n k} \delta(\phi-\psi),
\end{aligned}
$$

and similarly for the other cases. As a consequence a generic operator $\hat{A}$ may be written as

$$
\hat{A}=\sum_{n \in \mathbb{Z}} \int_{-\pi}^{\pi} \frac{d \phi}{2 \pi} \operatorname{Tr}\left[\hat{A} \hat{R}_{n}^{\dagger}(\phi)\right] \hat{R}_{n}(\phi) .
$$

For estimating HO operators one needs a recipe to obtain the expectation value of $\hat{R}_{n}(\phi)$. Using a resolution of identity in terms of phase vectors $\left|e^{i \phi}\right\rangle$

$$
\hat{\mathbb{I}}=\int_{-\pi}^{\pi} \frac{d \phi}{2 \pi}\left|e^{i \phi}\right\rangle\left\langle e^{i \phi}\right|
$$

one evaluates the traces as

$\operatorname{Tr}\left[\hat{\varrho} \hat{R}_{q}(\psi)\right]=\left\{\begin{array}{cc}\int_{-\pi}^{\pi} \frac{d \phi}{2 \pi} e^{-i q \phi}\langle\phi-\psi|\hat{\varrho}| \phi\rangle & q \geq 0 \\ \int_{-\pi}^{\pi} \frac{d \phi}{2 \pi} e^{i q(\phi+\psi)}\langle\phi|\hat{\varrho}| \phi+\psi\rangle & q \leq 0\end{array}\right.$.

Eq. 37 implies that the knowledge of $\left\langle\hat{R}_{n}(\phi)\right\rangle$ is equivalent to that of the density matrix in the phase representation.

\section{Quantum estimation in spin systems}

The recently born spin tomography [5, 4] allows to reconstruct the quantum state of a spin $s$ system. It employs measurements of the spin component in different directions, 
i.e. it uses as quorum the set of operators $\vec{S} \cdot \vec{n}$, where $\vec{S}$ is the spin operator and $\vec{n}$ a unit vector. Various different quorums may be constructed by exploiting different directions.

The easiest choice is to consider all possible directions. The procedure to derive the tomographic formulas for this quorum is analogous to the one employed in Sect. 3.1 for homodyne tomography. The reconstruction formula for spin tomography for the estimation of an arbitrary operator $\hat{A}$, using the measurement outcomes $m$ of the component of the spin in all directions $\vec{n} \doteq(\cos \varphi \sin \vartheta, \sin \varphi \sin \vartheta, \cos \vartheta)$, is

$$
\langle\hat{A}\rangle=\sum_{m=-s}^{s} \int_{\Omega} \frac{d \vec{n}}{4 \pi} p(m, \vec{n}) R[\hat{A}](m, \vec{n}),
$$

where $p(m, \vec{n})$ is the probability of obtaining the eigenvalue $m$ when measuring $\vec{S} \cdot \vec{n}$, $\hat{R}[\hat{A}](m, \vec{n})$ is the tomographic kernel for the operator $\hat{A}$, and $\Omega$ is the unit sphere. In this case the operators $\hat{C}$ of Eq. (何) are given by the set of projectors over the eigenstates $|m, \vec{n}\rangle$ of the operators $\vec{S} \cdot \vec{n}$ for all directions $\vec{n}$. Notice that it is a set of irreducible operators in the system Hilbert space $\mathcal{H}$. In order to find the dual basis $\hat{B}$, one must consider the unitary operators obtained by exponentiating the quorum, i.e. $\hat{D}(\psi, \vec{n})=\exp (i \psi \vec{S} \cdot \vec{n})$, which satisfy the bi-orthogonality condition (4). In fact, $\hat{D}(\psi, \vec{n})$ constitutes a unitary irreducible representation of the group $\mathrm{SU}(2)$, and the biorthogonality condition is just the orthogonality relations between the matrix elements of the group representation [9], i.e.

$$
\int_{R} d g \hat{D}_{j r}(g) \hat{D}_{t k}^{\dagger}(g)=\frac{V}{d} \delta_{j k} \delta_{t r},
$$

where $\hat{D}$ is a unitary irreducible representation of dimension $d, d g$ is the group Haar invariant measure, and $V=\int_{R} d g$. For $\mathrm{SU}(2)$, with the $2 s+1$ dimension unitary irreducible representation $\hat{D}(\psi, \vec{n})$, Haar's invariant measure is $\sin ^{2} \frac{\psi}{2} \sin \vartheta d \vartheta d \varphi d \psi$, and $\frac{V}{d}=\frac{4 \pi^{2}}{2 s+1}$. Thus, the bi-orthogonality condition is

$$
\frac{2 s+1}{4 \pi^{2}} \int_{\Omega} d \vec{n} \int_{0}^{2 \pi} d \psi \sin ^{2} \frac{\psi}{2}\left\langle j\left|e^{i \psi \vec{n} \cdot \vec{S}}\right| r\right\rangle\left\langle t\left|e^{-i \psi \vec{n} \cdot \vec{S}}\right| k\right\rangle=\delta_{j k} \delta_{t r},
$$

Using Eq. (40) and condition 1, it is immediate to write the spin tomography identity

$$
\hat{A}=\frac{2 s+1}{4 \pi^{2}} \int_{\Omega} d \vec{n} \int_{0}^{2 \pi} d \psi \sin ^{2} \frac{\psi}{2} \operatorname{Tr}\left[\hat{A} \hat{D}^{\dagger}(\psi, \vec{n})\right] \hat{D}(\psi, \vec{n}) .
$$

Notice the strict analogy between Eq. (41) and Glauber's formula (13), analogy which derives from the group symmetry that underlies both homodyne tomography (WeylHeisenberg group) and spin tomography (SU(2) group). In fact, both these tomographies may be derived in the domain of Group Tomography [5]. In order to obtain the reconstruction formula (38), one only has to take the expectation value of both members of Eq. (41) and to evaluate the expectation value trace on the eigenstates $|m, \vec{n}\rangle$ of $\vec{S} \cdot \vec{n}$. Thus, the explicit form of the tomographic kernel is obtained as

$$
R[\hat{A}](m, \vec{n})=\frac{2 s+1}{\pi} \int_{0}^{2 \pi} d \psi \sin ^{2} \frac{\psi}{2} \operatorname{Tr}\left[\hat{A} e^{-i \psi(\vec{S} \cdot \vec{n}-m)}\right] .
$$


As already anticipated, there are other possible quorums for spin tomography. For example, for spin $s=\frac{1}{2}$ systems, a self-dual basis for the operator space is given by the Pauli matrices and the identity $\left\{\frac{1}{\sqrt{2}} \hat{\sigma}_{x}, \frac{1}{\sqrt{2}} \hat{\sigma}_{y}, \frac{1}{\sqrt{2}} \hat{\sigma}_{z}, \frac{1}{\sqrt{2}} \hat{1}\right\}$. Hence, we would expect to find a tomographic identity where the above operators constitute a quorum. In fact, from the property $\hat{\sigma}_{\alpha} \cdot \hat{\sigma}_{\alpha^{\prime}}=\hat{1} \delta_{\alpha \alpha^{\prime}}\left(\alpha, \alpha^{\prime}=x, y, z\right)$, it is immediate to see that both the biorthogonality relation (4) and the trace condition (6) hold. The following reconstruction formula for an arbitrary $2 \times 2$ matrix $\hat{A}$ derives

$$
\langle\hat{A}\rangle=\sum_{m=-\frac{1}{2}}^{\frac{1}{2}} \sum_{\alpha=x, y, z} p\left(m, \vec{n}_{\alpha}\right) \operatorname{Tr}\left[\hat{A} \hat{\sigma}_{\alpha}\right] m+\frac{1}{2} \operatorname{Tr}[\hat{A}] .
$$

In the case of generic $s$ spin system, Weigert has shown [4] that it is possible, by choosing $(2 s+1)^{2}$ arbitrary directions for $\vec{n}$, to obtain (in almost all cases) a quorum of projectors $\left|s, \vec{n}_{j}\right\rangle\left\langle s, \vec{n}_{j}\right|\left(j=1, \cdots,(2 s+1)^{2}\right)$, where $\left|s, \vec{n}_{j}\right\rangle$ is the eigenstate pertaining to the maximum eigenvalue $s$ of $\vec{S} \cdot \vec{n}_{j}$. The search for the co-basis is done numerically, but since we have a discrete quorum, it is possible to use the procedure derived from Gram-Schmidt orthogonalization method, which is presented in Appendix B.

\section{Quantum state of a free particle}

Can we infer the state of a moving packet from position measurement in time? The answer is positive as can be rigorously proved using our condition (田). The probability density of the position of a free particle at the time $\tau$ is obtained from the selfadjoint operator

$$
\hat{R}(x, \tau)=e^{-i \hat{p}^{2} \tau}|x\rangle\langle x| e^{i \hat{p}^{2} \tau},
$$

where $|x\rangle$ are eigenstates of the position operator and $\hat{p}$ the momentum operator. We suppose for simplicity a particle with unit mass and use normalized unit $\hbar / 2=1$, such that the free Hamiltonian is given by $\hat{H}_{F}=\hat{p}^{2}$. The dual basis is constituted by the set of operator $\hat{R}(x, \tau)$ themselves, as follows from Eq. (4) and from the following relations $(|j\rangle, j=a, b, c, d$ denote position eigenstates)

$$
\begin{array}{rl}
\int_{\mathbb{R}} \int_{\mathbb{R}} & d x d \tau\langle a|\hat{R}(x, \tau)| b\rangle\langle c|\hat{R}(x, \tau)| d\rangle \\
& =\int_{\mathbb{R}} \int_{\mathbb{R}} d x d \tau e^{-i \tau\left(b^{2}-a^{2}+c^{2}-d^{2}\right)}\langle a \mid x\rangle\langle x \mid b\rangle\langle c \mid x\rangle\langle x \mid d\rangle \\
& =\int_{\mathbb{R}} \int_{\mathbb{R}} d x d \tau e^{-i \tau\left(b^{2}-a^{2}+c^{2}-d^{2}\right)} e^{i x(a-b+c-d)} \\
& =\delta(a-c) \delta(b-d) .
\end{array}
$$

Therefore, a generic free particle state can be written as

$$
\hat{\varrho}=\int_{\mathbb{R}} \int_{\mathbb{R}} d x d \tau p(x, \tau) \hat{R}(x, \tau)
$$


$p(x, \tau)=\operatorname{Tr}[\hat{\varrho} \hat{R}(x, \tau)]$ being the probability density of the particle to be at position $x$ at time $\tau$. Eq. (46) can be generalized to particle moving in arbitrary potential, for details see Ref. [15].

\section{Summary}

A unified approach to quantum state estimation has been presented. Some general conditions that guarantee that a set of observables is sufficient for the estimation were given. A constructive algorithm to derive dual basis, and therefore quantum estimators, is suggested for the finite dimensional case. Our framework allows to describe all known quantum tomographies (i.e. unbiased state estimation procedures) for single mode radiation field, trapped ions, vibrational states of molecules and spin systems. Moreover, using our characterizations, some new tomographic resolutions were derived. These may be used to implement novel estimation techniques.

\section{Appendix A. Liouville space formulation}

In this section we reformulate the general scheme of quantum estimation, given in Sect. 2, by making use of the properties of the linear space of operators acting on the system Hilbert space $\mathcal{H}$, i.e. the Liouville space $\mathcal{L}(\mathcal{H})$. This may results in a more convenient visualization of of our conditions 1 and 2, since customary Dirac notation can be used. The Liouville space is built starting from the set of operators of Hilbert-Schmidt type, which is itself a Hilbert space, with the scalar product

$$
\langle\hat{A} \mid \hat{B}\rangle \doteq \operatorname{Tr}\left[\hat{A}^{\dagger} \hat{B}\right] .
$$

Using Dirac formalism on the operator Hilbert space, we find that kets are the operators of $\mathcal{L}(\mathcal{H})$, i.e. $\hat{O} \doteq|\hat{O}\rangle$, while bras are obtained with the substitution $\operatorname{Tr}\left[\cdot \hat{O}^{\dagger}\right] \doteq\langle\hat{O}| \cdot$. The space of operators may be extended by considering non normalizable vectors in $\mathcal{L}(\mathcal{H})$. Using Dirac notation, we can rewrite all the formulas introduced so far. The generalized tomography formula (11) corresponds to the expansion of the vector $|\hat{A}\rangle$ on the non-orthogonal basis $|\hat{C}(x)\rangle$, that will be referred to as "spanning set", and on its dual $\langle\hat{B}(x)|(x \in \mathcal{X})$, i.e.

$$
|\hat{A}\rangle=\int_{\mathcal{X}} d x|\hat{C}(x)\rangle\langle\hat{B}(x) \mid \hat{A}\rangle .
$$

In this framework, conditions 1 and 2 represent respectively the identity resolution for the spanning set and the definition of the dual vectors $\langle\hat{B}(x)|$ of the set $|\hat{C}(x)\rangle$. In fact, 
we can readily rewrite Eqs. (4) and (6) as

$$
\begin{aligned}
& \int_{\mathcal{X}} d x|\hat{C}(x)\rangle\langle\hat{B}(x)|=\hat{\hat{1}}, \\
& \langle\hat{B}(x) \mid \hat{C}(y)\rangle=\delta(x, y) .
\end{aligned}
$$

The linear structure of the Liouville space provides a necessary and sufficient condition to verify that a set of operators is a quorum. A set of vectors $|\hat{C}(x)\rangle$, such that $\langle\hat{B}(x) \mid \hat{C}(y)\rangle=\delta(x, y) \forall x$, is a spanning set (with $\langle\hat{B}(x)|$ as dual set) iff the only operator $|\hat{O}\rangle \in \mathcal{L}(\mathcal{H})$ that is orthogonal to all $|\hat{C}(x)\rangle_{\mathrm{s}}$ is the null operator, i.e. iff the two equivalent conditions

$$
\langle\hat{O} \mid \hat{C}(x)\rangle=\operatorname{Tr}\left[\hat{O}^{\dagger} \hat{C}(x)\right]=0 \quad\langle\hat{B}(x) \mid \hat{O}\rangle=\operatorname{Tr}\left[\hat{B}^{\dagger}(x) \hat{O}\right]=0
$$

(for any $x \in \mathcal{X}$ ) imply that $\hat{O}=0$.

In addition to the reconstruction of operators acting on the system Hilbert space $\mathcal{H}$, one can extend the formalism also to the reconstruction of super-operators acting on the system operator space. A typical example is the Liouvillian super-operator that evolves the system density operator into a density operator. In fact, by introducing two resolutions of the identity in the operator space, one can express any super-operator $\hat{\mathrm{L}}$ in terms of its "matrix elements" $\langle\hat{B}(x)|\hat{\hat{L}}| \hat{C}(y)\rangle$ on a basis for $\mathcal{L}(\mathcal{H})$, i.e.

$$
\hat{\hat{\mathrm{L}}}=\int_{\mathcal{X}} d x \int_{\mathcal{X}} d y|\hat{C}(x)\rangle\langle\hat{B}(x)|\hat{\hat{\mathrm{L}}}| \hat{C}(y)\rangle\langle\hat{B}(y)| \text {. }
$$

By taking a basis $\{|n\rangle\}$ of $\mathcal{H}$, Eq. (A.6) rewrites as

$$
\begin{aligned}
& L_{m p}^{k l}=\langle k|\hat{\hat{\mathrm{L}}}[|m\rangle\langle l|]| p\rangle= \\
& \int_{\mathcal{X}} d x \int_{\mathcal{X}} d y \operatorname{Tr}[\hat{C}(x)|p\rangle\langle k|] \operatorname{Tr}[\hat{B}(y)|m\rangle\langle l|] \operatorname{Tr}[\hat{B}(x) \hat{\hat{\mathrm{L}}}[\hat{C}(y)]] .
\end{aligned}
$$

\section{Appendix B. Orthogonalization procedure}

Here we give an algorithmic procedure, usable in the case of finite quorums, to construct the set of dual operators $\left\langle\hat{B}_{n}\right|$ of the quorum $\left|\hat{C}_{n}\right\rangle$. Using the Gram-Schmidt orthogonalization method, one obtains a basis $\left|y_{k}\right\rangle$ from a complete set of vectors $\left|C_{k}\right\rangle$ (assume for simplicity that all $\left|C_{k}\right\rangle$ are non-zero and that in $\left\{\left|C_{k}\right\rangle\right\}$ there are no couples of proportional vectors):

$$
\left\{\begin{array}{c}
\left|y_{0}\right\rangle \doteq \frac{1}{N_{0}}\left|C_{0}\right\rangle \\
\left|y_{k}\right\rangle \doteq \frac{1}{N_{k}}\left(\left|C_{k}\right\rangle-\sum_{j=0}^{k-1}\left|y_{j}\right\rangle\left\langle y_{j} \mid C_{k}\right\rangle\right)
\end{array},\right.
$$

$\|$ Eq. (A.3) is readily obtained from Eq. (任 by introducing the identity super-operator, defined as $\hat{\hat{1}}[\hat{A}] \doteq \hat{A}$ for any $\hat{A} \in \mathcal{L}(\mathcal{H})$. In fact, by using the basis $\{|n\rangle\}$ for $\mathcal{H}$, we see that

$$
\langle k|\hat{\hat{1}}[|m\rangle\langle l|]| p\rangle=\delta_{m k} \delta_{l p} .
$$


where $N_{0} \doteq \|\left|C_{0}\right\rangle \|$ and $N_{k} \doteq \|\left|C_{k}\right\rangle-\sum_{j=0}^{k-1}\left|y_{j}\right\rangle\left\langle y_{j} \mid C_{k}\right\rangle \|$. Now, by writing the identity resolution

$$
\hat{1}=\sum_{k=0}\left|y_{k}\right\rangle\left\langle y_{k}\right| \equiv \frac{\left|C_{0}\right\rangle}{N_{0}}\left\langley _ { 0 } \left|+\sum_{k=1} \frac{1}{N_{k}}\left(\left|C_{k}\right\rangle-\sum_{j=0}^{k-1}\left|y_{j}\right\rangle\left\langle y_{j} \mid C_{k}\right\rangle\right)\left\langle y_{k}\right|,\right.\right.
$$

and using repeatedly Eq. (B.1) (expressing $\left|y_{j}\right\rangle$ of Eq. (‥2) in terms of the $\left|C_{n}\right\rangle$ s and reorganizing the terms) we can find the dual set $\left\langle B_{n}\right|$ as

$$
\begin{aligned}
& \left\langle B_{0}\right|=\frac{\left\langle y_{0}\right|}{N_{0}}-\frac{\left\langle y_{0} \mid C_{1}\right\rangle\left\langle y_{1}\right|}{N_{0} N_{1}}+\left(-\frac{\left\langle y_{0} \mid C_{2}\right\rangle}{N_{0} N_{2}}+\frac{\left\langle y_{0} \mid C_{1}\right\rangle\left\langle y_{1} \mid C_{2}\right\rangle}{N_{0} N_{1} N_{2}}\right)\left\langle y_{2}\right|+\cdots \\
& \left\langle B_{1}\right|=\frac{\left\langle y_{1}\right|}{N_{1}}-\frac{\left\langle y_{1} \mid C_{2}\right\rangle\left\langle y_{2}\right|}{N_{1} N_{2}}+\left(-\frac{\left\langle y_{1} \mid C_{3}\right\rangle}{N_{1} N_{3}}+\frac{\left\langle y_{1} \mid C_{2}\right\rangle\left\langle y_{2} \mid C_{3}\right\rangle}{N_{1} N_{2} N_{3}}\right)\left\langle y_{3}\right|+\cdots
\end{aligned}
$$

Unfortunately no such a general procedure appears to exist for the case of a continuous spanning set. Many cases, though, satisfy the conditions (9) and (10), and hence we can write $\langle\hat{B}(x)|=(|\hat{C}(x)\rangle)^{\dagger}$.

\section{Acknowledgments}

This work has been partially supported by INFM through project PAIS-1999-TWIN.

\section{References}

[1] H. K. Lo et al., Introduction to Quantum Computation and Information, World Scientific (1998).

[2] G. M. D'Ariano, P. Kumar, and M. F. Sacchi, Phys. Rev. A 61, 013806 (2000)

[3] T. Opatrny and D. -G. Welsch, Prog. Opt. vol. XXXIX, 63 (1999).

[4] S. Weigert, Phys. Rev. Lett. 84, 802 (2000).

[5] G. M. D'Ariano, Phys. Lett. A 268151 (2000).

[6] K. Vogel, H. Risken, Phys. Rev. A 40, 2847 (1989).

[7] G. M. D'Ariano, C. Macchiavello, M. G. A. Paris, Phys. Rev. A 50, 4298 (1994); G. M. D'Ariano, U. Leonhardt, M. Paul, Phys. Rev. A 52 R1801 (1995).

[8] G. M. D'Ariano, "Measuring quantum states", in Quantum Optics and the Spectroscopy of Solids, ed. by T. Hakioğlu and A.S. Shumovsky, Kluwer Academic Publishers (1997), p. 175.

[9] F. D. Murnaghan, The theory of group representation, pg. 216, Johns Hopkins Press (1938).

[10] G. M. D’Ariano, S. Mancini, V. I. Manko, and P. Tombesi, Q. Opt. 8, 1017 (1996)

[11] K. Banaszek and K. Wodkievicz, Phys. Rev. Lett. 76, 4344 (1996).

[12] T. Opatrny and D. -G. Welsch, Phys. Rev. A, 55, 1462 (1997).

[13] D. Leibfried et al., Phys. Rev. Lett. 77, 4281 (1996).

[14] S. Wallentowitz, W. Vogel, P. L. Knight, Phys. Rev. A 59, 531 (1999).

[15] Th. Richter, A. Wünsche, Acta Phys. Slov. 46, 487 (1996). 